\title{
Localized low-frequency vibrational modes in glasses
}

\author{
H.R. Schober and Brian B. Laird* \\ Institut für Festkörperforschung, Forschungszentrum Jülich GmbH, Postfach 1913, D-5170 Jülich 1, Federal Republic \\ of Germany
}

(Received 29 April 1991)

\begin{abstract}
Using a larger data set and more detailed analysis, we continue our examination of the zerotemperature vibrational spectrum of a glass of soft spheres by computer simulation. As in our previous study [Phys. Rev. Lett. 66, 636 (1991)], a normal-mode analysis shows clearly the existence of (quasi-)localized modes at the low-frequency end of the vibrational spectrum. The modes are found to be localized around atoms whose neighborhood structure differs significantly from the average glass environment and is reminiscent of that associated with interstitial defects in crystals. The effective masses of these modes range upward from 10 atomic masses.
\end{abstract}

\section{INTRODUCTION}

Although both are elastic solids, glasses and crystals of the same material exhibit strikingly different lowtemperature properties. At temperatures below $1 \mathrm{~K}$, the heat capacity of an amorphous solid is significantly greater than that of the corresponding crystal and exhibits a nearly linear variation with temperature rather than the $T^{3}$ dependence predicted by the Debye model which describes the low-temperature properties of the crystalline phase. ${ }^{1}$ This anomalous behavior of the glassy phase can be explained by assuming that the dominant low-frequency contribution to the density of states of the glass is due to two-level tunneling states. ${ }^{2,3}$

This now-standard tunneling model is unable, however, to account for the anomalous behavior of the same glassy materials at intermediate temperatures between 1 and $10 \mathrm{~K}$, where the heat capacity is no longer linear in $T$. In this region, the heat capacity, $C_{v}$ is still considerably larger than the crystal value and a plot of $C_{v} / T^{3}$ versus $T$ shows a large peak that is either absent or much less pronounced in the crystal. ${ }^{4}$ This peak seems to be a universal phenomenon of glasses and is due to the presence at intermediate frequency of a significant fraction of low-energy states that are neither tunneling states nor sound waves. ${ }^{5}$ Recent neutron scattering experiments have shown these additional states to be soft harmonic vibrations that are localized to about 10 or more atoms. ${ }^{6}$

It is plausible to assume that both the two-level states and these soft harmonic vibrations have a similar structural origin. A theory exploiting this idea was developed by Karpov et al. ${ }^{7}$ They describe both the two-level systems and the harmonic vibration states by soft anharmonic potentials for some effective reaction coordinate. Fitting this model to the experimental data for various glasses gives between 20 and 70 for the number of atoms participating in the vibration. ${ }^{8}$ Even though such models are successful in providing a consistent interpretation of the experimental data over the entire low-temperature range, they, like the experiments they attempt to describe, have not answered the fundamental questions as to the physical nature and precise structural origin of either the tunneling or soft vibrational modes. A microscopic understanding of these soft modes could give some important insight into the physics of the glassy phase and, perhaps, into the glass transition itself.

In a recent letter ${ }^{9}$ we presented results of computer simulations that clearly showed the existence of localized low-frequency vibrational modes in a simple soft-sphere glass. Our results also showed that the neighbor shell around atoms that participate strongly in these soft localized vibrations is more compressed and contains slightly fewer atoms than the corresponding environment of an average particle. Thus, these modes can be viewed as being associated with "defects" in the glass. In this followup paper we repeat these calculations using a larger data base to give improved statistics and perform a much more detailed analysis of the microscopic origin of these modes.

The approach we follow is similar to the study by Nagel et $a{ }^{10}{ }^{10}$ of vibrational localization in a modified LennardJones glass, except that they concerned themselves only with the high-frequency modes. Low-frequency localized vibrations were found in a computer generated model for amorphous silicon by Biswas et al. ${ }^{11}$ but interpretation of the results is complicated by their use of a different potential to calculate the vibrational properties than was used to create the glass structure.

\section{LOCALIZATION IN GLASSES}

The idea of disorder-induced localization of excitations is most familiar from studies on electronic systems. ${ }^{12}$ Here, as the disorder is increased, the states at both the high- and low-energy ends of the density of states become localized first, while those in the center remain extended. For the case of phonons, however, true localization in a disordered system occurs only for those states at the high-frequency end of the normal-mode distribution, ${ }^{13}$ because in any elastic medium there are always acoustic modes at low frequency, which are by nature extended. Any low-frequency localized mode would then hybridize 
with these, destroying the strict local nature of the vibration. However, these hybrid modes retain their localized character, e.g., with regard to scattering properties. Such states are termed as resonant or quasilocalized. These low-frequency quasilocalized vibrational modes are very important for the low-temperature properties.

The concepts of "localized" and "(quasi-)localized" vibrations are well known in the phonon theory of defects in crystals. ${ }^{14}$ The former denotes a vibration with a frequency outside the continuum of lattice frequencies. Such a vibration cannot couple to the lattice modes and its eigenvector decays exponentially with distance. These modes occur commonly either for very light impurities or for defects that cause large lattice strains such as selfinterstitial atoms. In a glass, this type of vibration will be found in the high-frequency tail of the normal-mode spectrum. For low frequencies within the lattice continuum, where the host density of states is very low, resonant defect vibrations are possible. Such resonant modes are similar to localized modes in the usual definition and are often referred to as quasilocalized low-frequency modes. Like true localized vibrations, the eigenvector of such a mode is also localized to the defect and a few neighbors, but generally not as strongly, and does not decay exponentially. ${ }^{15}$ Evidence for the existence of such resonant modes at low frequencies in addition to the usual high-frequency localized modes was found by computer simulation for self-interstitials in fcc metals by Dederichs et $a l .{ }^{16}$ The existence of these low-frequency modes was verified experimentally by neutron scattering. ${ }^{17}$ They are a common feature of interstitials in random alloys. ${ }^{18}$

The amount of localization can be quantified in terms of the normalized eigenvectors, $e_{\alpha}^{j}$, of the vibrational states, where the index $j$ runs over all atoms in the sample and $\alpha$ labels the Cartesian coordinate. The motion of the mode is described by

$$
u_{\alpha}^{j}(t)=\frac{f(t) e_{\alpha}^{j}}{M_{j}^{1 / 2}}
$$

where $M_{j}$ is the mass of atom $j$ and $u_{\alpha}^{j}(t)$ is its displacement in the $\alpha$ direction. Without loss of generality we assume atom 1 to have the largest displacement. Within the harmonic approximation the kinetic energy of the mode is then

$$
=\frac{1}{2}[\dot{f}(t)]^{2}=\frac{1}{2} \sum_{\alpha} M_{\mathrm{eff}}\left(\dot{u}_{\alpha}^{1}\right)^{2}
$$

where the effective mass, $M_{\text {eff }}$ is defined as

$$
M_{\mathrm{eff}} \equiv \frac{M_{1}}{\sum_{\alpha}\left(e_{\alpha}^{1}\right)^{2}}=M_{1} /\left|\mathbf{e}^{1}\right|^{2}
$$

$M_{\text {eff }}$ is thus a measure for the number of atoms which effectively carry the kinetic energy of the vibrational mode. Note that, this definition of the effective mass is valid for not too large systems. For an infinite system the effective mass of a resonant mode can be defined exactly in terms of the vibrational Green's function. ${ }^{15}$ Our definition is the limit for not too large finite systems. By this defini- tion the effective mass measures the fraction of the total vibrational spectrum of the atom which is contained in the resonant mode.

For localized and quasilocalized modes, $M_{\mathrm{eff}}$ is small and system-size independent, whereas for extended modes, it scales with the number of particles.

An alternative definition of localization often used is the participation ratio

$$
p=\left(N \sum_{j}\left|\mathbf{e}^{j}\right|^{4}\right)^{-1} .
$$

For extended modes, $p$ is of order unity. For localized or quasilocalized modes, it will scale inversely with the system size.

The participation ratio can be used to define a participation mass by

$$
M_{\mathrm{part}}=p N \text {. }
$$

The two masses $M_{\text {eff }}$ and $M_{\text {part }}$ are only equal in a few limiting cases. For example, if we assume the vibrational amplitudes of the first $N_{s}$ atoms $\left(N_{s} \ll N\right)$ are equal while the amplitudes on the other atoms are of order $1 / N$ we get

$$
\left|\mathbf{e}^{j}\right|^{2}=\left\{\begin{array}{l}
m / M_{\mathrm{eff}}, \text { if } j \leq N_{s} \\
\left(1-N_{s} m / M_{\mathrm{eff}}\right) /\left(N-N_{s}\right), \text { otherwise. }
\end{array}\right.
$$

In this simple case we get

$$
M_{\text {part }} \approx M_{\text {eff }}^{2} /\left(N_{s} m\right) \text {. }
$$

The participation mass is always larger than the effective mass, the more the larger the fraction of the amplitude in the long-range tails. In the limits of the most localized and delocalized modes we have $M_{\text {eff }}=M_{1} \rightarrow p=1 / N$ and $M_{\text {eff }}=N M_{1} \rightarrow p=1$. For self-interstitials in fcc metals one finds for the low-frequency localized modes that $M_{\text {eff }} \approx 4$ or 5 times $M_{1}$ and for the high-frequency ones about half that value. The effective mass is sensitive to the defect-defect interaction-two interstitials clustered together will raise $M_{\text {eff }}$ about a factor of 2 . The effective mass for tunneling transitions of these defects was found to be similar to the one for the low-frequency modes. ${ }^{19}$

In a glass with its large distortions we expect to find both types of localized vibrational modes. One cannot expect to find isolated defectlike structures, but there will always be strong interaction between such centers. Correspondingly, we expect the single-defect effective masses to be something like a lower limit of the ones in glasses.

\section{CREATION OF GLASS CONFIGURATIONS}

We perform a computer simulation for a system of soft spheres interacting with an inverse sixth-power potential:

$$
u(r)=\epsilon\left(\frac{\sigma}{r}\right)^{6}+A\left(\frac{r}{\sigma}\right)^{4}+B
$$


To simplify the computer simulation and normal-mode analysis, the potential was cut off at $r / \sigma=3.0$, and then shifted by a polynomial, $A r^{4}+B$, where $A=2.54 \times 10^{-5} \epsilon$ and $B=3.43 \times 10^{-3} \epsilon$ were chosen so that the potential and the force are zero at the cutoff. This form of the shifting function was chosen so that its effect is negligible near $r / \sigma=1.0$. Quantities such as the pressure and average potential energy will be changed by a few percent as a result of this truncation, but any changes in the equilibrium structure will be small. At any rate, our interest is in the existence and characterization of lowfrequency localized vibrational modes in general, and not in a quantitative description for any specific potential.

The inverse sixth-power potential was selected because it is a well-studied theoretical model that qualitatively mimics many of the structural and thermodynamic properties of bcc forming metals including the existence, in its bcc crystal form, of very soft shear modes, ${ }^{20}$ and it was hoped that this property would be reflected in a high concentration of low-frequency resonant modes in the glass. As evidence of the universality of the phenomena discussed here, we also found low-frequency resonant modes in one- and two-component Lennard-Jones systems, but at such a low concentration that the collection of any reasonable statistics would have been very difficult.

We produce our glass configurations by quenching a well-equilibrated liquid configuration of 500 soft spheres (1024 for the larger system) produced via constantenergy molecular-dynamics (MD) simulation with cubic periodic boundary conditions at a density $\rho \sigma^{3}=1.0$, and temperature $k T / \epsilon \approx 0.54$ (about 2.5 times the melting temperature at this density $\left.{ }^{21}\right)$. For the simulation we used the velocity-Verlet algorithm ${ }^{22}$ with a time step of 0.02 -in units of $\left(m \sigma^{2} / \epsilon\right)^{1 / 2}$. The liquid is first quenched within the MD simulation by velocity rescaling to a reduced temperature of about 0.04 . The quench rate was about $0.25 k /\left(m \sigma^{2} \epsilon\right)^{1 / 2}$.

An estimation of the glass-transition temperature was obtained by calculating the diffusion constant $\mathrm{D}$ of the system at this density as a function of the reduced temperature using the relation

$$
D=\lim _{t \rightarrow \infty} \frac{1}{6 t}<|\mathbf{R}(0)-\mathbf{R}(t)|^{2}>,
$$

where $\mathbf{R}(t)$ is the time-dependent position vector of a particle and $\langle\cdots\rangle$ denotes a configurational average. The diffusion constant so calculated (shown in Fig. 1) is very well fit by a fractional power law

$$
D=A\left(T-T_{0}\right)^{\alpha},
$$

with $A=0.191, \alpha=1.21$, and $T_{0}=0.085$. That such a fitting function so well describes the diffusion data is consistent with the predictions of mode-coupling theory. ${ }^{23}$ The temperature $T_{0}$ can be taken as a lower limit for the glass transition temperature, since below $T_{0}$ diffusive motion is effectively frozen out. The above after-quench temperature corresponds then to roughly half this $T_{0}$.

After the MD quench, each sample was aged for 1200 further MD time steps to stabilize the potential energy. It should be noted that at this temperature the glass is not stable indefinitely against crystallization, but the average

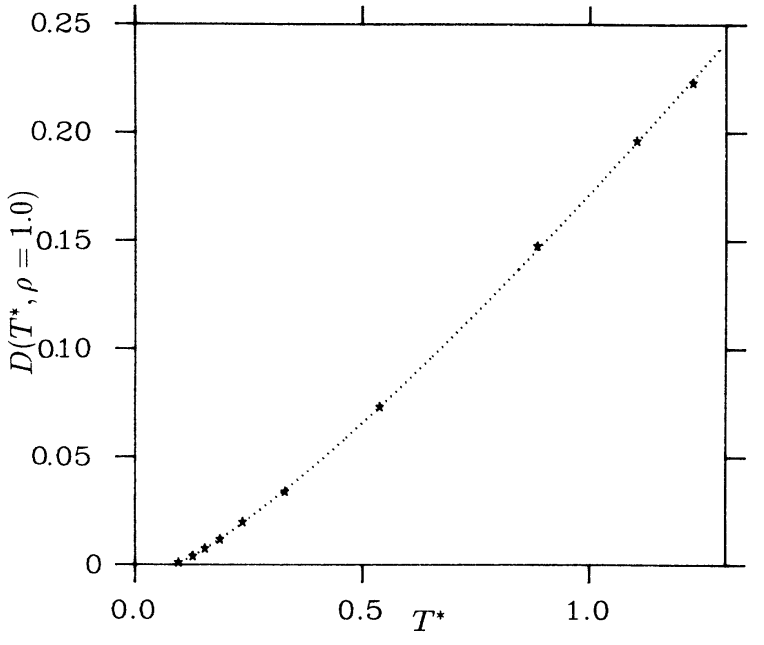

FIG. 1. Self-diffusion constant of the $\left(1 / r^{6}\right)$-soft-sphere system as a function of the reduced temperature $(N=500$, $\left.\rho \sigma^{3}=1.0\right)$. The dashed line is a power-law fit (see text).

time for such a nucleation to occur is an order of magnitude greater than the times considered here. Each system is then quenched to zero temperature using a combination steepest-descent/conjugate-gradient algorithm. ${ }^{24}$ The resulting potential energy equals roughly the one of the fcc crystal of the same density with about $1.5 \%$ Frenkel defects. In all, 60 different 500-atom configurations and thirty 1024-atom glass configurations were created in this way and analyzed, thus the total number of normal modes was approximately the same in both sample sizes. These numbers of configurations represent

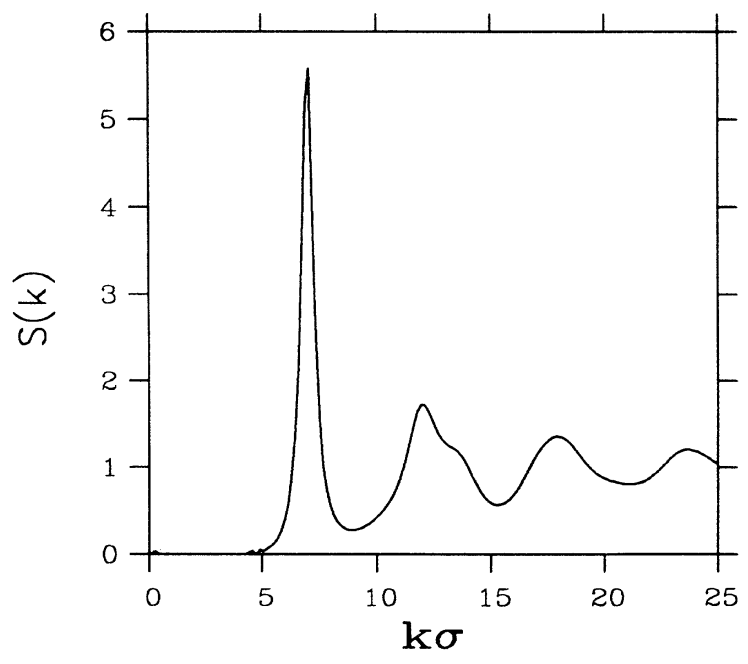

FIG. 2. The configurationally averaged structure factor for our inverse sixth-power glass at zero temperature. 
a factor of 2 (10) increase for the smaller (larger) system over those used in our previously reported calculation. ${ }^{9}$

To illustrate the glassy nature of our zero-temperature sample and to aid comparison of this model glass to real materials, we have also plotted (Fig. 2) the configurationally averaged structure factor $S(k)$ for our system. This function was obtained by Fourier transforming the configurationally averaged pair correlation function $g(r)$, using an integral equation method due to Verlet ${ }^{25}$ to correct for errors due to the truncation of $g(r)$.

\section{SPECTRA AND LOCALIZATION OF VIBRATIONAL MODES}

For each glass configuration the force-constant matrix (using periodic boundary conditions) was calculated and diagonalized using standard EISPACK routines ${ }^{26}$ on the CRAY X-MP computer to yield the $3 N(N=500$ or 1024) normal-mode frequencies and eigenvectors. The participation ratio and effective mass for each mode were then calculated. These quantities were sorted by frequency into bins of width 0.03 and averaged over all configurations to yield a frequency profile. For the participation ratio, this frequency profile is plotted in Fig. 3 for both the 500- and 1024-atom configuration sets.

The most obvious feature of this plot is the dramatic drop of the participation ratio at both the high- and lowfrequency ends of the spectrum. The drop at the high end of the spectrum is due to the usual high-frequency localized modes. We argue that the low-participation ratios at the low-frequency end of the plot are due to the presence of resonant or quasilocalized modes. One indicator of the localized nature of these modes is that, for the very lowest frequencies, the participation ratios for the 1024-

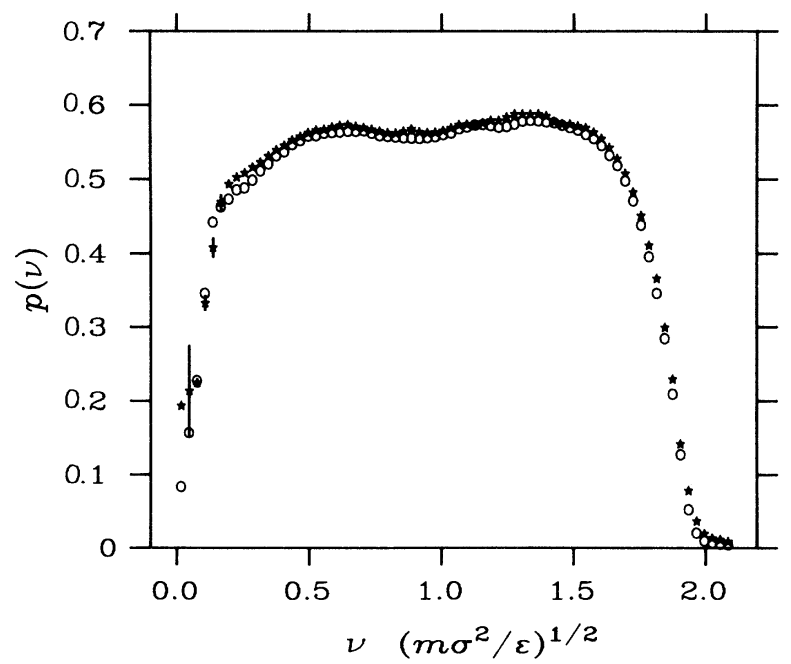

FIG. 3. The configurationally averaged participation ratio plotted as a function of frequency for both the 500-atom (stars) and 1024-atom (circles) systems. The error bars are an upper estimate of the statistical error, obtained by coarse graining.

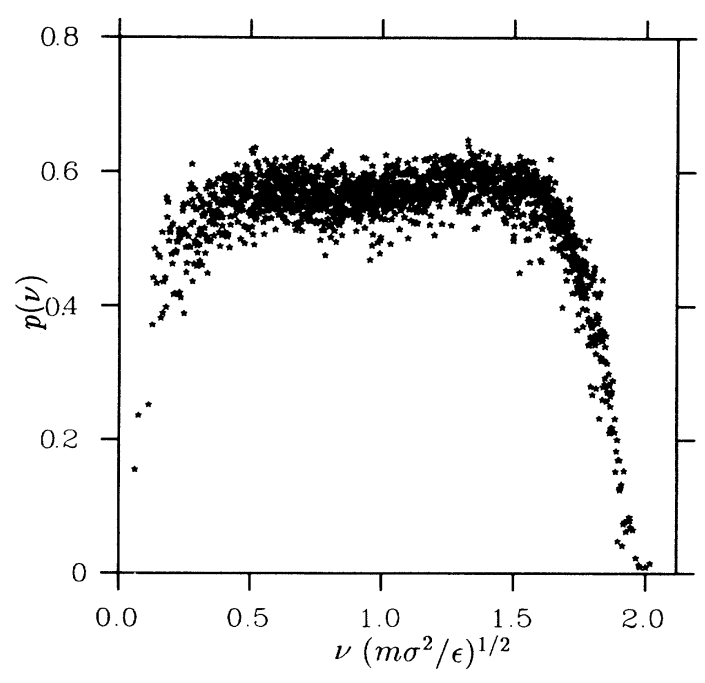

FIG. 4. Participation ratios $(p)$ of the individual modes in one configuration plotted against the mode frequencies.

atom system are about a factor of 1.4 to 2 smaller than those for the 500-atom system. The factor of about 2 expected from (4) is an upper bound which is reduced by two effects. First in a larger system the extended modes reach down to lower frequencies, the minimal possible $q$ value scales with $N^{-1 / 3}$. Second, more important, in a larger system there is more likelihood of interaction of soft modes. Such an interaction causes an increase of $p$. Further down we will show that such an interaction is indeed observed.

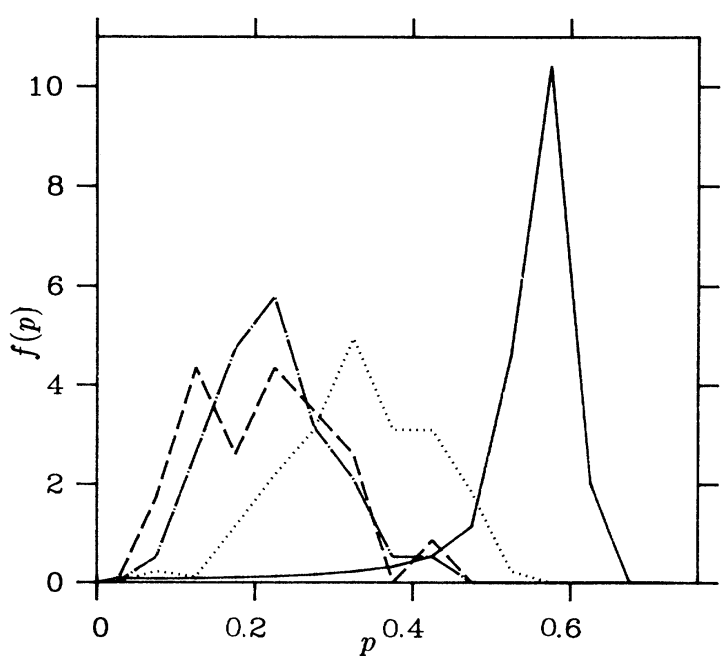

FIG. 5. Spectrum of the participation ratios $p$ averaged over 60 configurations $(N=500)$ for all modes (solid line), modes with $\nu \leq 0.06$ (dashed line), modes with $0.06<\nu \leq$ 0.09 (dash-dotted line), and modes with $0.9<\nu \leq 0.12$ (dotted line), respectively. 
The error bars were calculated by dividing the configurations into six sets of ten (five for the larger system) and calculating $\langle p(\nu)\rangle$ for each and the standard deviation in the remaining six averages. Despite this coarse graining, this error estimate is still influenced by the inherent scatter in $p(\nu)$ and represents only a conservative upper bound of the actual statistical error. An idea of this inherent spread in the participation ratios for a given frequency can be seen in Fig. 4 where the participation ratios for all modes in a specific 500-atom configuration are plotted. Averaged over all configurations one can in the usual way define a spectrum $f(p)$ of the $p$ values for a given frequency. Figure 5 shows such spectra for three frequency ranges where soft modes are found and also averaged over all frequencies. The total distribution of $p$ is strongly peaked around 0.58 with a long tail to small $p$ values. This tail is mainly given by the localized high-frequency modes. For the strongly localized modes $(p<0.2) f(p)$ is nearly constant except for a cutoff at the low end. The spectrum of $p$ for the low-frequency modes is substantially broader, but clearly shifted to lower $p$ values.

In terms of the effective mass, defined in Eq. (3), we get the same general picture. In Fig. 6 we plot the average effective mass as a function of frequency for both the small and large systems. The details of averaging process and error analysis are the same as for the calculation of $p(\nu)$. Except for a size-dependent change in scale, the plot for the effective mass is nearly identical in shape to the corresponding participation ratio curve; therefore the results of our study will be nearly independent of the choice of localization criterion. Comparing Figs. 3 and 6 one sees that for $N=500$ a participation ratio of $p=0.25$ corresponds on average to an effective mass

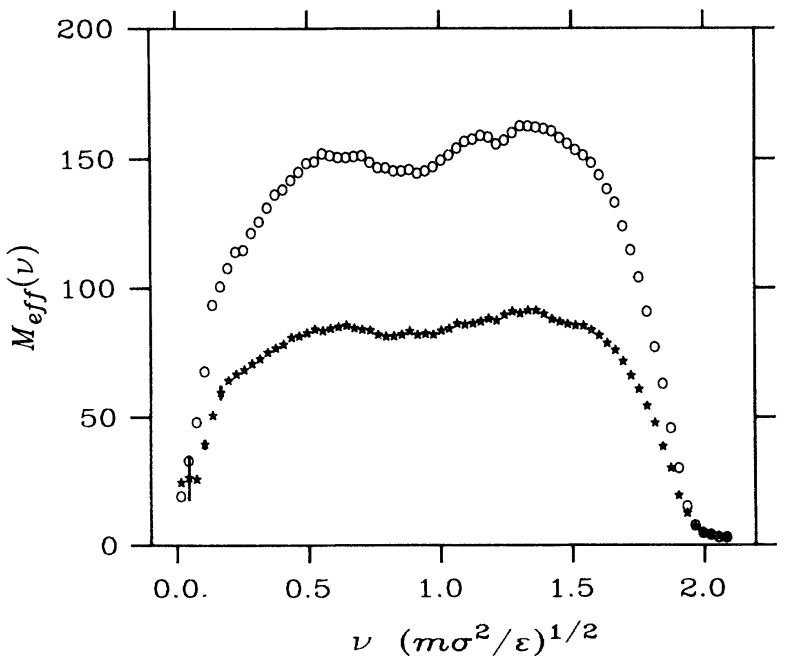

FIG. 6. The configurationally averaged effective mass plotted as a function of frequency for both the 500-atom (stars) and 1024-atom (circles) systems. The error bars are an upper estimate of the statistical error, obtained by coarse graining.

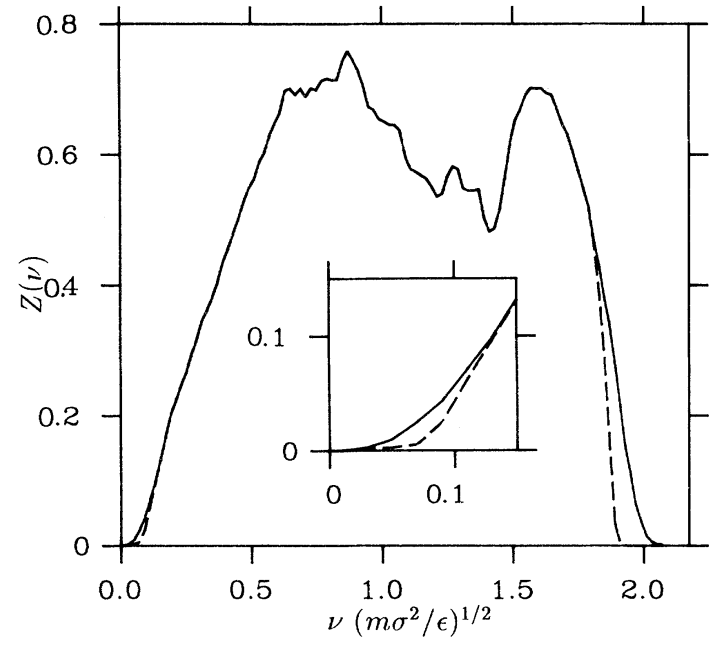

FIG. 7. The configurationally averaged vibrational density of states as a function of frequency for (a) all modes (solid line) and (b) all modes $(N=500)$ with $p>0.25$ (dashed line): The inset is an enlargement of the low-frequency tail of these curves.

of $M_{\mathrm{eff}} \approx 25$. Please note that there is no linear scaling between $M_{\text {eff }}$ and $p$ or $M_{\text {part }}$.

Figure 7 shows the configurationally averaged density of states for this system along with the same quantity with all modes (97 in all) subtracted out for which $p_{c} \leq 0.25$ - out of the 60 configurations only 7 contained no such modes. This cutoff was arbitrarily chosen to yield a clear distinction between localized and nonlocalized modes; however, we have checked that the qualitative features of our result do not change for cutoffs in the range $0.15 \leq p_{c} \leq 0.35$. The total spectrum has the shape typical for a simple glass. ${ }^{27}$ On both the highand low-frequency sides one sees localization. The weak tail $\left[Z(\nu) \propto \nu^{2}\right]$ of the extended modes for $\nu \rightarrow 0$ is suppressed due to the limited system size. The localized modes at high frequencies comprise a fraction of about $10^{-2}$ of the total spectrum. The fraction of low-frequency localized modes is an order of magnitude smaller. Nevertheless the resonant modes make a significant contribution to the density of states at very low frequencies, and, thus, would be expected to profoundly affect the low-temperature behavior of the glass.

\section{STRUCTURAL ORIGIN OF SOFT LOCALIZED MODES}

Some insight into the structural nature of these resonant modes can be obtained by examining the twoparticle radial distribution function, $g(r)$. In Fig. 8 we plot this function calculated by averaging over all available zero-temperature glass configurations. Also, for each low-frequency resonant mode (for both $p_{c} \leq 0.25$ and $p_{c} \leq 0.35$ ), the particle with the largest displacement was determined, and $g(r)$ calculated, using this particle as the 


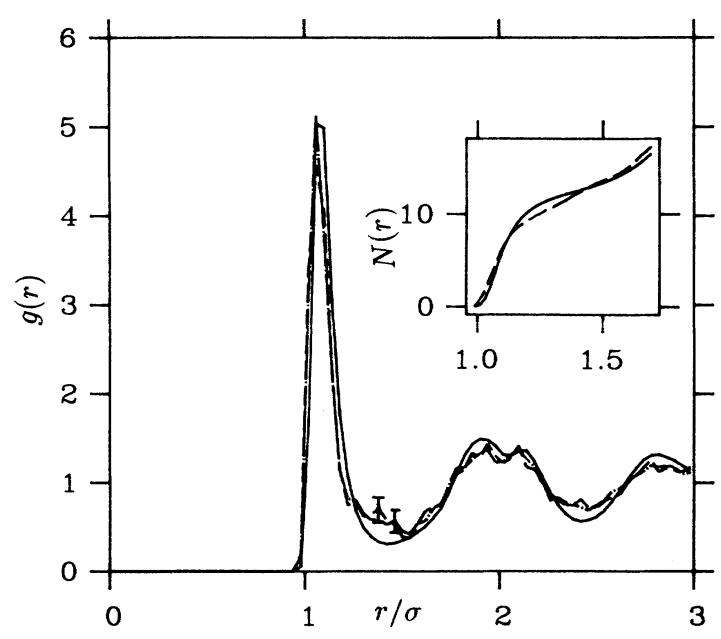

FIG. 8. The configurationally averaged two-particle radial distribution function, $g(r)$ measured (a) for the full system (solid line), (b) using as the central atom only those particles with the largest displacement for some mode $(N=500)$ with $p<0.25$ (dashed line), and (c) same as (b) but $p<0.35$ (dash-dotted line). The inset shows the integrated form of this quantity-the total radial particle number, $N(r)$. The error bars are estimates of the statistical error (see text) for $p<0.25$.

central atom. Averaged over all the resonant modes, this quantity is also plotted in Fig. 8. [The error bars were calculated as for $p(\nu)$.] From these plots we can see that the nearest-neighbor shell is more compressed and less dense for the resonant-mode central atom than that for the average particle. This first-shell particle deficiency in the resonant $g(r)$ is made up for by a small peak just outside the first peak, after which the integrated particle number (see the inset to Fig. 8) for the two displayed distribution functions are nearly identical. The plots for $p_{c}=0.25$ and $p_{c}=0.35$ are hardly discernible in Fig. 8 and also the plot for $p_{c}=0.15$ (not shown) looks identical within the statistical error. The difference of the $g(r)$ of the resonating atoms to the average $g(r)$ is, therefore, insensitive to the chosen value of $p_{c}\left(0.15 \leq p_{c} \leq 0.35\right)$. It is also insensitive to the number of configurations used in the averaging process, and is, therefore, a real effectnot just a statistical fluctuation. Thus, the existence of the quasilocalized soft modes cannot be derived from the average structural properties of the glass, as is done, for example, in the effective medium approximation. ${ }^{28}$

Another measure of the spatial extent of the resonant modes is the degree of localization of the kinetic energy. Defining the central atom (atom 1) as the atom that has for a given mode the largest amplitude (as we did in the calculation of $g(r)$, we can calculate the fraction of the kinetic energy residing within a sphere of radius $r$ about this central atom as

$$
\frac{E_{\mathrm{kin}}^{i}(r)}{E_{\mathrm{kin}}^{i}}=\sum_{\left|\mathbf{R}^{m}-\mathbf{R}^{1}\right|<r}\left|\mathbf{e}^{m}(i)\right|^{2},
$$

where $\mathbf{e}^{m}(i)$ is the projection of the eigenvector for mode $i$ on atom $m$. For an extended mode this quantity should grow as $r^{2}$ since the amplitude of a given mode on each atom should be approximately constant. A resonant mode should give a slower rise. In Fig. 9 the configurational average of $E_{\text {kin }}(r)$ contained in one periodicity volume is plotted for $p_{c}=0.25, p_{c}=0.35$, and for the overall average. As predicted the average $E_{\text {kin }}$ increases as $r^{2}$ whereas for the localized modes the increase is more rapid. In a sphere of, e.g., $r=4 \sigma$ there is for the quasilocalized modes twice the fraction of the kinetic energy than for the average mode.

We have seen that there is a strong indication that the localized soft modes originate from some local structural differences. This should reflect itself in the dynamics of the single atoms. Let us first look at the Einstein modes of the atoms, i.e., the vibrations of the single atoms with their neighbors at rest. Figure 10 shows the spectrum of these modes averaged over all configurations of 500 atoms each. As to be expected from the shape of the pair correlation function, the spectrum shows a broad distribution of Einstein frequencies. However, the Einstein spectrum shows no tail to either high or low frequencies. No information on the nature of the localized modes can be obtained from this spectrum.

More detailed information is contained in the local spectra of the atoms. We define the local vibrational spectrum of atom $m$ in the standard way as

$$
Z^{m}(\nu)=\sum_{i}\left|\mathbf{e}^{m}(i)\right|^{2} \delta\left(\nu-\nu_{i}\right),
$$

where the sum is over all modes $i$ and $e$ denotes the eigenvector. This local spectrum weights each eigenmode with its squared amplitude on atom $m$. For an ideal crystal it

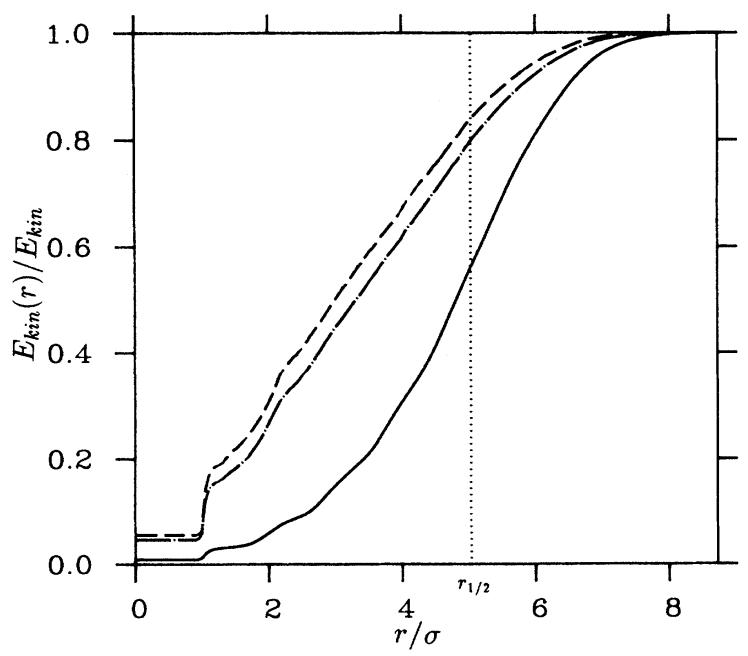

FIG. 9. Fraction of the kinetic energy of the vibrational modes contained in a sphere of radius $r$ : (a) average over all modes (solid line), (b) average over soft modes with $p<0.12$ ( $N=1024$ ) (dashed line), and (c) as (b) but $p<0.17$ (dashdotted line). $r_{1 / 2}$ denotes half the periodicity length. 


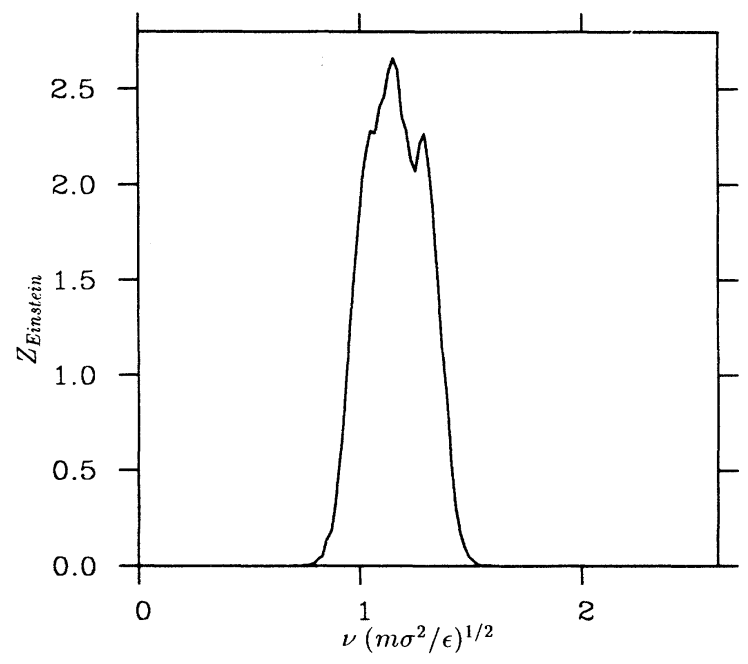

FIG. 10. Configurationally averaged spectrum of Einstein frequencies.

is identical to the usual spectrum. The second $\nu$ moment of the local spectrum gives the Einstein frequency. Figure 11 shows the local spectra of three atoms averaged over the three space directions. The local spectrum of an atom which does not participate in localized vibrations (a) looks similar to the average spectrum (Fig. 7). In (b)

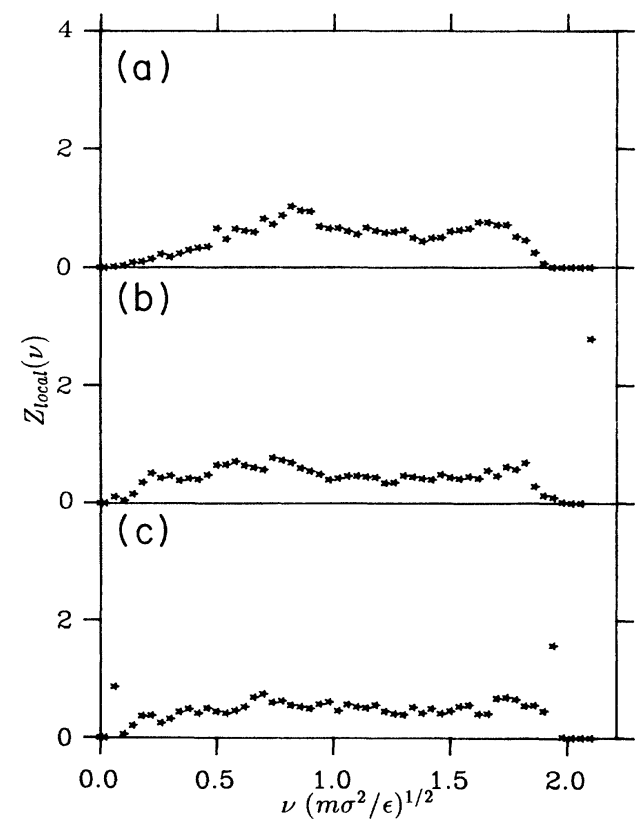

FIG. 11. Local spectra (see text for definition) of selected atoms: (a) atom participating mainly in extended modes, (b) atom participating in a high-frequency localized mode, and (c) atom participating in both high- and low-frequency localized modes.
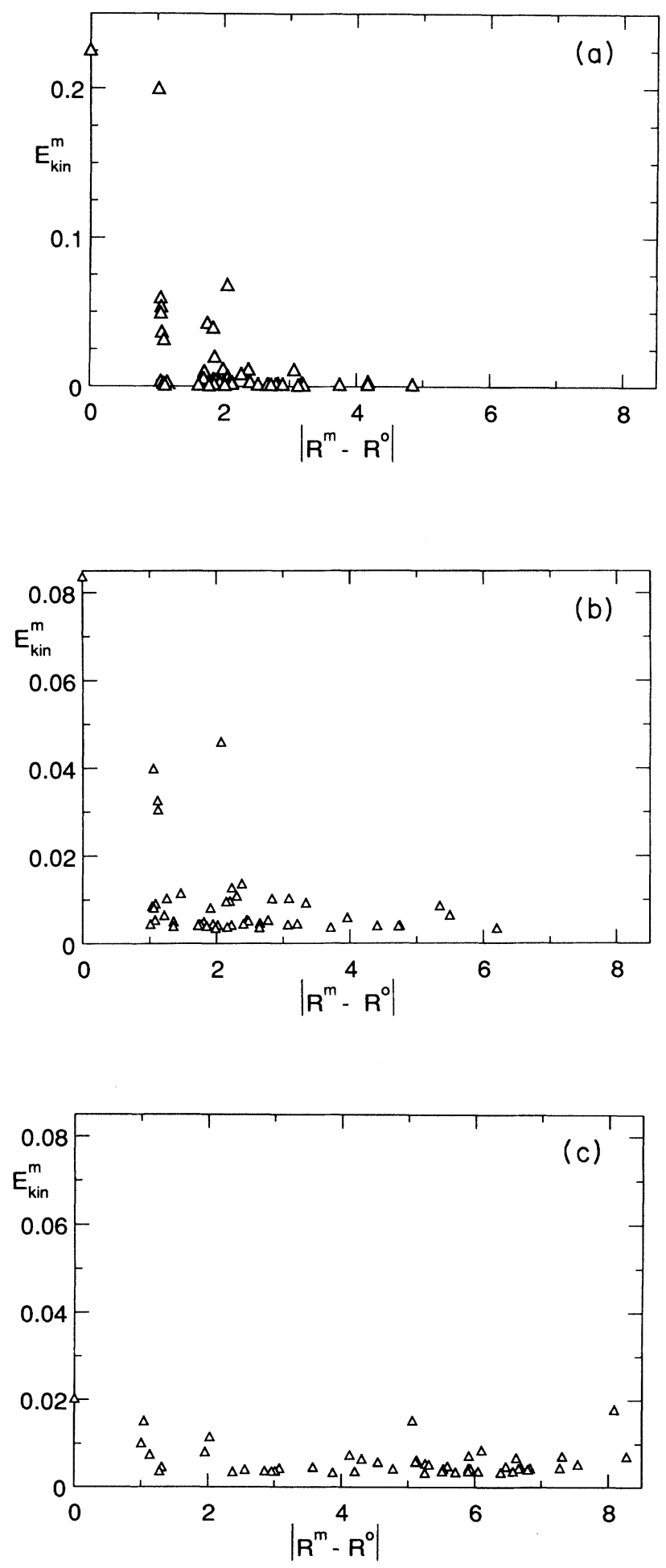

FIG. 12. Contributions, $E_{\text {kin }}^{m}$, of the single atoms to the total kinetic energy of localized modes as function of the distance of the atom to the central atom of the mode $(\mathrm{N}=500)$ : (a) high-frequency localized mode $\left(\nu=2.02, M_{\text {eff }}=4.5\right.$, $p=0.01$ ), (b) noninteracting quasilocalized (resonant) mode ( $\left.\nu=0.06, M_{\text {eff }}=12, p=0.06\right)$, and (c) interacting quasilocalized mode $\left(\nu=0.09, M_{\text {eff }}=49, p=0.27\right)$. Note the different energy scales. 
we show the spectrum of an atom participating in a highfrequency localized vibration but not in a low-frequency one. Apart from the localized vibration above $\nu=2$ the spectrum shows a broad distribution of modes, perhaps slightly shifted to lower frequencies. Figure 11(c) finally shows the spectrum of a mode participating in a low-frequency localized mode. The spectrum shows simultaneously a high-frequency localized mode. Such spectra are familiar from self-interstitial defects in crystals. The explanation is there that the high-frequency localized modes are caused by the local compression of the neighborhood of the interstitial. Frequently but not always the local configuration is such that a vibration is possible which does not strain the compressed bonds. Such a vibration can become soft (resonant mode) or even unstable. This scenario is a possible explanation for the observed spectrum and for the hard Einstein spectrum where the "soft" directions are compensated by the hard ones. The atoms (or atom clusters) where the low-frequency modes are centered would then be a subset of the atoms participating in high-frequency modes. Whether this picture is true for all low-frequency modes cannot be determined with certainty at the moment since the high effective masses of these modes mean low contributions to the relevant local spectra. The pair correlation functions for both sets of atoms show the same compression of the first neighbor shell. As noted before $g(r)$ of the centers of the resonant modes has additional intensity in the first minimum of the average $g(r)$, Fig. 8 . This feature is absent for $g(r)$ averaged over the centers of all high-frequency localized modes. It might be the signature of the structures allowing resonant modes.

\section{DISCUSSION AND CONCLUSION}

Our investigation shows clearly the existence of localized low-frequency modes in accordance with recent experiments and theoretical conjectures. These lowfrequency modes are found in much lower concentrations $\left(10^{-3}\right)$ than their high-frequency counterparts. As is to be expected, their localization is also much weaker. The most localized modes are found to have effective masses of about 10 to 30 atomic masses. The frequencies of these modes are well below $\frac{1}{10}$ of the maximum vibrational frequency. There is a broad distribution of effective masses. By its very nature computer simulation can only give results on the low-mass side of the distribution. The transition between localized and delocalized modes cannot be determined unambiguously due to the limited system size. There seems to be a shift of the mean effective mass with frequency but, especially at the low-frequency end, the data do not suffice to extract the exact relation between mass and frequency. High- and low-frequency localized modes are found centered around the same atoms.
The two-particle radial distribution function, $g(r)$, for those atoms that maximally contribute to the lowfrequency localized modes indicates a structural difference to that of the average glass: the nearest-neighbor distance is reduced and about one atom is pushed from the nearest-neighbor shell into the region where the average $g(r)$ has its first minimum. Whereas the compression of the first shell is also found for the high-frequency localized modes the latter property of $g(r)$ is not present for these. It, therefore, might be caused by some additional feature only present in that subset of the configurations leading to high-frequency localization which also produced low-frequency localized modes.

At least partially, the width in the distribution of effective masses or participation ratios is due to clustering effects. It is well known from the studies of point defects in crystal that these tend to cluster. We have seen that the soft modes occur around atoms which have a surrounding different from the average and hence in some way constitute defects. During the time the glass is cooled down from the glass transition temperature to $T=0 \mathrm{~K}$ some local rearrangements (relaxations) are still possible. The high "defect" density present in our soft-sphere glass makes it plausible that the defects will agglomerate. Such agglomeration will regularly lead to an increase of the participation ratio and effective mass. That agglomeration has occurred in our simulation can be seen from the eigenvectors of the modes. The quantity $E_{\mathrm{kin}}^{m}=\left|\mathrm{e}^{m}(i)\right|^{2}$ is the fraction of the kinetic energy of mode $i$ on atom $m$. To illustrate different possible situations we have for three typical modes plotted in Fig. 12 the 50 largest terms of $E_{\mathrm{kin}}^{m}$ as function of the distance from the central atom. For noninteracting localized modes this quantity is centered around the central atom. This is observed for the most localized modes, Figs. 12(a) and 12(b). For less localized modes we find in some cases the kinetic energy of one mode to be centered around two or even three well-separated atoms [Fig. 12(c)], which then participate in two or three localized modes. We interpret this as interaction of these modes. If the centers of two modes are close neighbors the distinction between one or two centers is no longer clear. This interaction of defects explains also why there is a quantitative difference in the average value of the participation of the soft modes for different quenching procedures. A study of this interplay of modes will be important for the understanding of low temperature relaxations.

\section{ACKNOWLEDGMENTS}

We are grateful for many stimulating discussions with U. Buchenau. This material is based on work supported by the North Atlantic Treaty Organization under a Grant awarded to B.L.

\footnotetext{
* Present address: Department of Chemistry, University of Utah, Salt Lake City, UT 84112.

${ }^{1}$ R.C. Zeller and R.O. Pohl, Phys. Rev. B 4, 2029 (1971).

${ }^{2}$ P.W. Anderson, B.I. Halperin, and C.M. Varma, Philos.
}

Mag. 25, 1 (1972).

${ }^{3}$ W.A. Phillips, J. Low Temp. Phys. 7, 351 (1972).

${ }^{4}$ R.O. Pohl and E.T. Swartz, J. Non-Cryst. Solids 76, 117 (1985). 
${ }^{5}$ S. Hunklinger and A.K. Raychoudhuri, Prog. Low Temp. Phys. 9, 267 (1986).

${ }^{6}$ U. Buchenau, H.M. Zhou, N. Nücker, K.S. Gilroy, and W.A. Phillips, Phys. Rev. Lett. 60, 1368 (1988).

${ }^{7}$ V.G. Karpov, M.I. Klinger, and F.N. Ignat'ev, Zh. Eksp. Teor. Fiz. 84, 760 (1983) [Sov. Phys. JETP 57, 439 (1983)]

${ }^{8}$ U. Buchenau, Yu. Galperin, V. Gurevich, and H.R. Schober, Phys. Rev. B 43, 5039 (1991).

${ }^{9}$ B.B. Laird and H.R. Schober, Phys. Rev. Lett. 66, 636 (1991).

${ }^{10}$ S.R. Nagel, A. Rahman, and G.S. Grest, Phys. Rev. Lett. 53, 368 (1984).

${ }^{11}$ R. Biswas, A.M. Bouchard, W.A. Kamitakahara, G.S. Grest, and C.M. Soukoulis, Phys. Rev. Lett. 60, 2280 (1988).

${ }^{12}$ D.J. Thouless, in Ill-Condensed Matter, edited by R. Balian, R. Maynard, and G. Toulouse (North-Holland, Amsterdam 1979)

${ }^{13}$ S. John, H. Sompolinski, and M.J. Stephen, Phys. Rev. B 27, 5592 (1983).

${ }^{14}$ A.A. Maradudin, E.W. Montroll, G.H. Weiss, and I.P. Ipatova, Theory of Lattice Dynamics in the Harmonic Approximation, Solid State Physics Suppl. 3 (Academic, New York, 1971).

${ }^{15}$ P.H. Dederichs and R. Zeller, in Point Defects in Metals II, Springer Tracts in Modern Physics Vol. 87 (Springer-Verlag, Berlin, 1980).
${ }^{16}$ P.H. Dederichs, C. Lehmann, and A. Scholz, Phys. Rev. Lett. 21, 1130 (1973).

${ }^{17}$ R. Urban, P. Ehrhart, W. Schilling, H.R. Schober, and H. Lauter, Phys. Status Solidi (B) 144, 287 (1987).

${ }^{18}$ P. Ehrhart, K.H. Robrock, and H.R. Schober, in Physics of Radiation Effects in Crystals, edited by R.A. Johnson and A.N. Orlov (North-Holland, Amsterdam, 1986).

${ }^{19}$ H.R. Schober and A.M. Stoneham, Phys. Rev. B 26, 1819 (1982).

${ }^{20}$ W.G. Hoover, S.G. Gray, and K.W. Johnson, J. Chem. Phys. 55, 1128 (1971).

${ }^{21}$ W.G. Hoover, D.A. Young, and R. Grover, J. Chem. Phys. 56, 2207 (1972).

${ }^{22}$ W.C. Swope, H.C. Anderson, P.H. Berens, and K. R. Wilson, J. Chem. Phys. 76, 637 (1982).

${ }^{23}$ U. Bengtzelius, W Götze, and A. Sjölander, J. Phys. C 17, 5915 (1984)

${ }^{24}$ R. Fletcher and C.M. Reeves, Comput. J. 7, 149 (1964).

${ }^{25}$ L. Verlet, Phys. Rev. 165, 201 (1968).

${ }^{26}$ B.T. Smith, J.M. Boyle, J.J. Dongarra, B.S. Garbow, Y. Ikebe, V.C. Klema, and C.B. Moler, Matrix Eigensystem Routines-EISPACK Guide (Springer-Verlag, Berlin, 1976).

${ }^{27}$ J.-B. Suck and H. Rudin, in Glassy Metals II, edited by H. Beck and H.-J. Güntherodt (Springer-Verlag, Berlin, 1983).

${ }^{28} \mathrm{~W}$. Schirmacher and M. Wagener, in Dynamics of Disordered Materials, edited by D. Richter, A.J. Dianoux, W. Petry, and J. Texeira (Springer-Verlag, Berlin, 1980). 